\title{
Mesenchymal Stem Cells Decrease Tunnel Widening of Anterior Cruciate Ligament Reconstruction in Rabbit Model
}

\author{
Chang-Ich Hur ${ }^{1}$, Hyeon-Wook Ahn ${ }^{1}$, Jong-Keun Seon ${ }^{1}$, Eun-Kyoo Song ${ }^{1}$, Ga-Eon Kim² \\ ${ }^{1}$ Center for Foint Disease, Chonnam National University Hwasun Hospital, Hwasun, Korea \\ ${ }^{2}$ Department of Pathology, Chonnam National University Medical School, Gwangju, Korea
}

Background and Objectives: The study investigated the effect of mesenchymal stem cells (MSCs) or fibrin glue on tunnel widening after anterior cruciate ligament (ACL) reconstruction compared with biologic free control without any biologic agents in the rabbit model.

Methods and Results: ACL reconstructions were performed in 18 New Zealand white rabbits. All animals were divided into 3 groups according to the following reconstruction conditions and euthanized 12 weeks postoperatively for radiologic and histologic analyses. Thirty-two knees (control group=10; fibrin group=11; MSCs group $=11$ ) were finally evaluated. On micro-CT scan, mean femoral tunnel widening on oblique-sagittal image was $0.7 \pm 0.4 \mathrm{~mm}$ in the control group, $0.22 \pm 0.1 \mathrm{~mm}$ in the fibrin group and $0.25 \pm 0.1 \mathrm{~mm}$ in the MSCs group $(\mathrm{p}=0.001)$. Fibrin group and MSCs group showed significant differences compared with control group $(p=0.002,0.002)$. Mean tibial tunnel widening on oblique-sagittal image was $0.76 \pm 0.5 \mathrm{~mm}, 0.27 \pm 0.1 \mathrm{~mm}$ and $0.29 \pm 0.2 \mathrm{~mm}$ in the control, fibrin and MSCs group. Fibrin and MSCs group showed significant differences compared with control group $(p=0.017,0.014)$. Hounsfield Units (HU) were not significantly different between 3 groups $(p>0.05)$. Histological analysis revealed that the architecture of graft in the MSCs group featured hypercellularity and compact collagen deposit.

Conclusion: ACL reconstruction using MSCs seemed decrease tunnel widening in rabbit model. Further study with large animals is required to confirm efficacy on decreasing tunnel widening.

Keywords: Anterior cruciate ligament, Mesenchymal stem cell, Tunnel widening, Rabbit model

\section{Introduction}

Anterior cruciate ligament (ACL) injuries are common in sports and it may result in symptoms such as move-

Received: March 20, 2018, Revised: March 23, 2018,

Accepted: November 8, 2018, Published online: December 31, 2018 Correspondence to Jong-Keun Seon

Center for Joint Disease, Chonnam National University Hwasun Hospital, 160 Ilsim-ri, Hwasun-eup, Hwasun 58128, Korea

Tel: +82-62-379-7676, Fax: +82-62-379-7681

E-mail: seonbell@chonnam.ac.kr

(c) This is an open-access article distributed under the terms of the Creative Commons Attribution Non-Commercial License (http://creativecommons.org/ licenses/by-nc/4.0/), which permits unrestricted non-commercial use, distribution, and reproduction in any medium, provided the original work is properly cited.

Copyright (c) 2019 by the Korean Society for Stem Cell Research ment dysfunction, knee instability, meniscus damage and early post-traumatic osteoarthritis $(1,2)$. Because of the poor regenerative potential of implanted graft, ACL reconstruction often results in the failure (3-5). The success of ACL reconstruction depends on many factors, including the initial mechanical properties of the graft $(6,7)$, positioning of the femoral and tibial tunnel $(8,9)$, fixation method $(10,11)$ and the postoperative rehabilitation. In addition, the biologic behavior of the graft is the one of the most important factors determining the mechanical properties of the grafts (12).

Improvement of early and secure graft integration to the bone can enable allow early and aggressive rehabilitation after ACL reconstruction. With the advanced knowledge of ACL healing and the development of biological strategies, many biologic agents have been introduced and ag- 
gressively developed in recent years. These include platelet-rich plasma $(13,14)$ growth factors $(13,15)$, and stem cell therapy (16-19). Among these agents, MSCs derived from bone marrow (bMSCs) have demonstrated promising potential in promoting graft osteointegration and graft healing (16-18). As well, the clinical application of mesenchymal stem cells (MSCs) has been explored as a way of enhancing the tissue regeneration in many orthopedic fields. MSCs are multipotent cells that can differentiate into variable cell types, including osteoblasts, chondrocytes, adipocyte and tenocytes (20-22), and are being investigated extensively in the enhancement of healing of tendon injuries and improvement of ACL reconstruction $(23,24)$. Fibrin glue is a formulation of fibrinogen and thrombin that is used to create a fibrin clot (25). Fibrin glue promotes repair of various connective tissues including cartilage and plays essential role in carrier of stem cells in ACL reconstruction in animal models $(26,27)$.

This study investigated three groups (control, fibirin, and MSCs group) and we conducted radiologic assessment with Quantum GX $\mu \mathrm{CT}$ imaging system for exact measurement of bone density, tunnel diameters, and tunnel lengths. To our knowledge, there were no studies which investigated three groups for bone density, tunnel widening and length with micro-CT. The purpose of the present study was to investigate the effect of MSCs or fibrin glue on tunnel widening after ACL reconstruction compared with biologic-free control using radiologic and histologic evaluations in a rabbit model.

\section{Materials and Methods}

\section{Animal and experimental design}

Eighteen healthy, adult female New Zealand white rabbits weighing 3,000 to 3,500 g underwent bilateral ACL reconstruction using an extensor digitorum longus tendon autograft harvested from the same surgical site. The physical conditions of all knees were carefully monitored during the experimental period. All experimental animal procedures were performed in strict accordance with our Institutional Animal Care and Use Committee. All knees were distributed into three groups according to use the biologic agent; control group $(n=12)$ without any biologic agent, fibrin group $(n=12)$ and MSCs group $(n=12)$ with stem cell and fibrin.

\section{Preparation of MSCs}

Bone marrow aspiration was carried out from the iliac crest of the rabbits in the control group as previously described $(25,26)$. Bone marrow $(1 \mathrm{ml})$ was collected and put in complete Dulbecco's Modified Eagle Medium (DMEM-lg, Gibco, USA) containing 10\% fetal bovine serum and cephazolin $1 \mathrm{~g} / \mathrm{ml}$. The sample was washed twice with DMEM-lg and centrifuged at 2,000 rpm for $6 \mathrm{~min}$. The supernatant was removed and the precipitating component was suspended in $10 \mathrm{ml}$ of stem cell growth medium. The stem cells were counted and plated (1.0 to $1.5 \times 10^{7}$ cells) in a $100 \mathrm{~mm}$ dish. Cultures were incubated at $37^{\circ} \mathrm{C}$ in an atmosphere $5 \% \mathrm{CO} 2$. After three days, nonadherent cells were removed when the medium was replaced with fresh medium. Once the colonies of MSCs reached $70 \sim 80 \%$ confluence, adherent cells were obtained from the flask with $0.05 \%$ trypsin. Homogenous MSCs were obtained after 2 weeks of culture. Passage two MSCs (P-2) were used.

\section{Surgical technique}

Each animal was then anesthetized with ketamine (35 $\mathrm{mg} / \mathrm{kg}$ ) and positioned in supinen. Both knees of each rabbit were shaved, scrubbed with betadine and covered with a sterile drape exposing only the unilateral knee. After midline incision, a medial para-patellar approach was performed and then the native ACL and extensor digitorum longi (EDL) tendon were identified (28) and the length was measured (Fig. 1A). The EDL tendon was harvested to a length of $20 \mathrm{~mm}$ or more and was kept immersed in sterile saline until implantation (Fig. 1B). After excision of the native ACL at its femoral and tibial origins, a $2 \mathrm{~mm}$-diameter bone tunnel was made at the footprints of the native ACL on the tibia and lateral femoral condyle (Fig. 1C, D). The reproducible and accurate tunnel positioning in relation to the native ACL footprint was necessary to achieve loading conditions similar to those of native knee joint. The free end of each EDL graft secured with 3-0 vicryl. In the fibrin group, $2 \mathrm{~mm}$ of fibrin glue (TISSEEL Kit, IMMUNO AG Vienna, Austria) was injected to the end of graft and MSCs embedded in fibrin glue by mixing was injected in the MSCs group. A total of $1 \times 10^{6}$ bone marrow stem cell with $1 \mathrm{ml}$ fibrin glue were injected in the end of graft and bone tunnel in MSCs groups. In fibrin group, a total of $2 \mathrm{ml}$ fibrin glue was injected in graft and tunnel. The prepared graft was passed through the tibial and femoral tunnels (Fig. 1E). The gap between the graft and the tunnel was filled with each biologic agent. A total of $1 \times 10^{7}$ stem cells with $1 \mathrm{ml}$ fibrin glue were injected to the end of graft and filled into the tunnel in MSCs groups. After extra-osseous postscrews were inserted near the tunnel orifices, the end of each implanted graft was anchored to screws using sutures (Fig. 1F). The incision was closed in layers with the ar- 

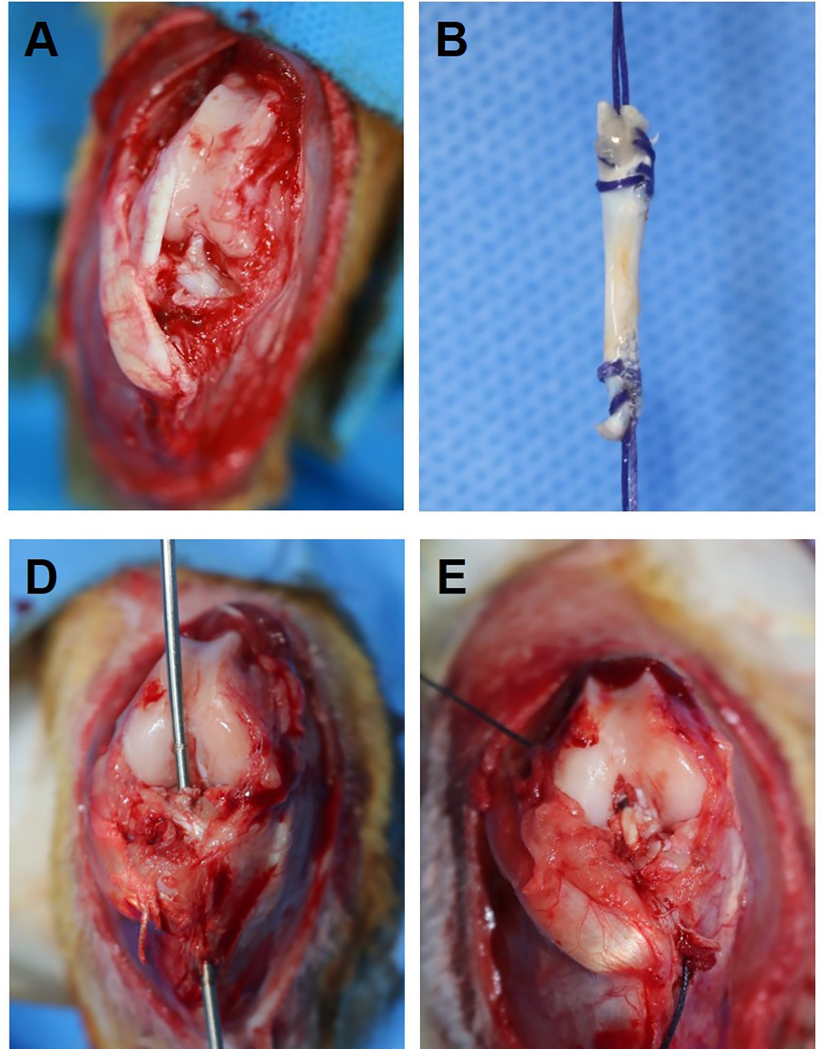

throtomy and subcutaneous layers. For initial stability, the postoperative knee was maintained in extension by an elastic bandage. Then the contralateral knee was operated on using the same method. All animals were euthanized at 12 weeks postoperatively for histologic analysis and for the evaluation of tunnel widening using micro-CT analysis.

\section{Radiologic analysis}

The knee joint was obtained by amputating at femoral and tibial shaft after euthanasia. CT imaging was performed using a Quantum GX $\mu \mathrm{CT}$ imaging system (PerkinElmer, Waltham, MA, USA). The X-ray source was set to levels of $90 \mathrm{kV}$ and $88 \mathrm{~mA}$ with a field of view of $72 \mathrm{~mm}$ with a slice thickness of $0.144 \mathrm{~mm}$. The scanning time was $14 \mathrm{~min}$ in a $360^{\circ}$ rotation. A matrix size of $512 \times 512 \times 512$ was then used to reconstruct using $3 \mathrm{D}$ Viewer, existing software within the Quantum GX. Following scanning, image segmentation was performed by Analyze software (AnalyzeDirect, Overland Park, KS, USA). To calculate the tunnel widening, the oblique-coronal and oblique-sagittal plane were obtained parallel to the tunnel (Fig. 2). The tunnel diameter was measured on $2 \mathrm{~mm}$ proximal and distal from the femoral and tibial articular surface. The tunnel widening was defined as differ-
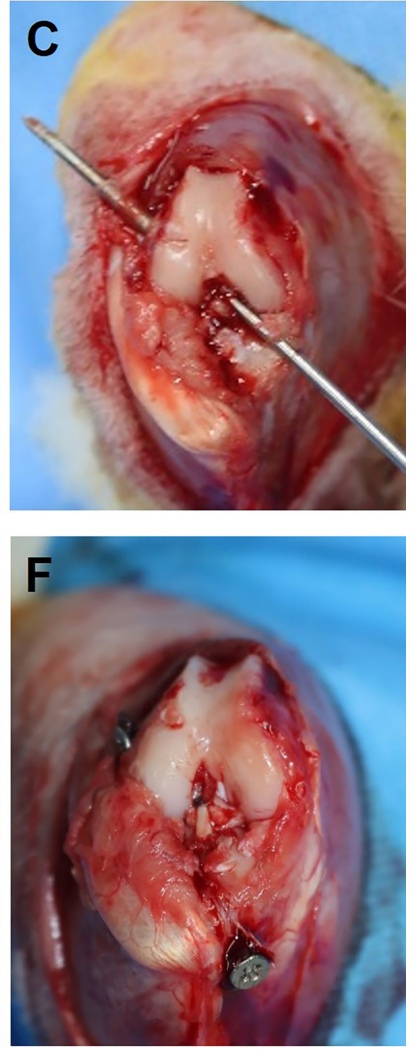

Fig. 1. Surgical process of $A C L$ reconstruction in rabbit model. (A) The native $\mathrm{ACL}$ and extensor digitorum longus were identified after medial para-patellar arthrotomy. (B) The EDL tendon with $2 \mathrm{~mm}$ diameter and $25 \mathrm{~mm}$ length was harvested. (C, D) Femoral and Tibial tunnel were made using $2 \mathrm{~mm}$ drill at footprints of native ACL. (E) The harvested tendon was passed through the femoral and tibial tunnel and (F) was tethered with suture to the screw inserted into the bone.

ence between measured diameter on CT scanning and drilled hole diameter $(2 \mathrm{~mm})$.

Then, Raw CT values were converted to Hounsfield Unit (HU) using intensity values of dry air was $-1,000$ $\mathrm{HU}$ and that of a water was $0 \mathrm{HU}$. To define each 1.5 $\mathrm{mm} \times 1.5 \mathrm{~mm}$ squared specific regions of interest (ROI) within the rabbit knees, the oblique plane images of the bone tunnel $2 \mathrm{~mm}$ proximal and distal from the femoral and tibial articular surface were analyzed using Quantum GX software. HUs were measured in each ROI in the tunnel and around tunnel in cancellous bone. All radiographic measurements were performed by two blinded observers not participating in surgery.

\section{Histologic analysis}

After euthanasia, each knee was carefully stripped from the surrounding soft tissues except from the autograft itself (Fig. 3). For histopathologic examination of the knee joints, each sample was fixed in 10\% buffered formalin. The specimens were decalcified in a decalcifying agent (Calci-Clear Rapid, National Diagnostics, GA) for 12 hours, cut along the grafted tendon in the oblique-sagittal direction, and embedded in paraffin. The paraffin block were sectioned at a $3 \mu \mathrm{m}$ thickness using a microtome (Leica Microsystems, Inc, Wetzlar, Germany) and each 

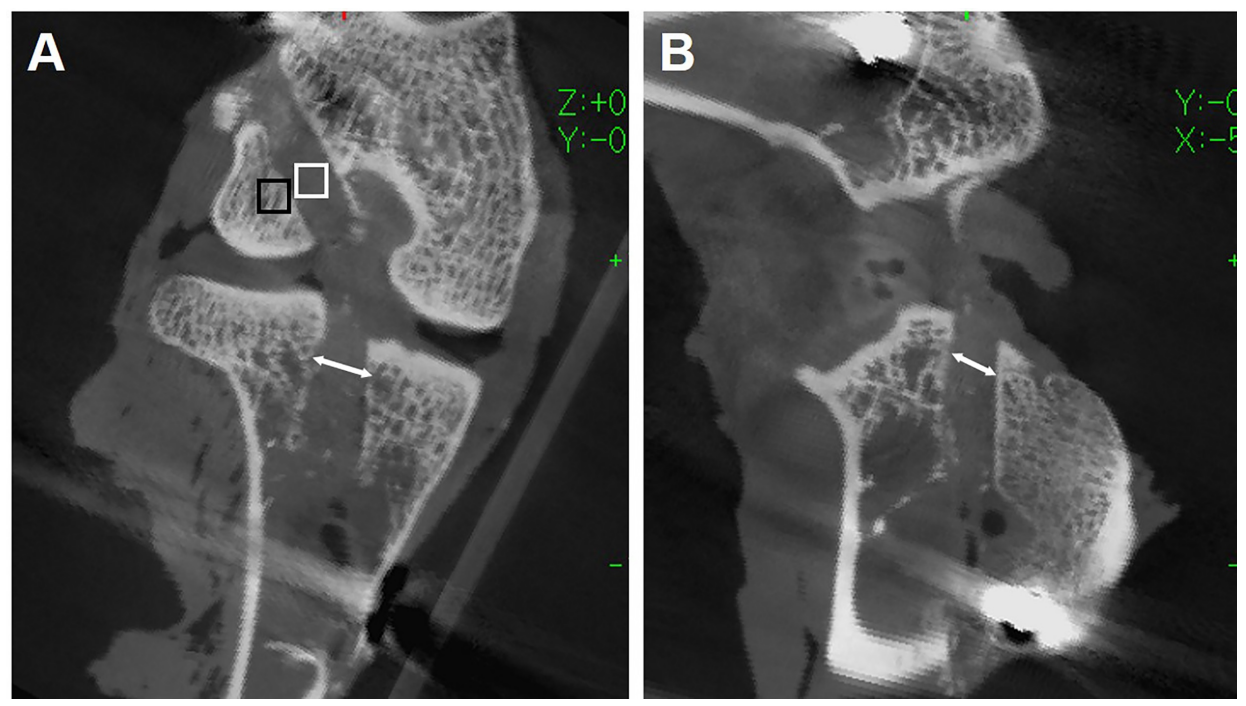

Fig. 2. CT image measuring tunnel widening and Hounsfield units (HUs). The tunnel diameter (white arrow) were calculated on the oblique-coronal (A) and oblique-sagittal image (B) $2 \mathrm{~mm}$ distal from the femoral and tibial articular surface. On the same distance from articular surface, HUs were measured in regions of interest $(1.5 \mathrm{~mm} \times 1.5 \mathrm{~mm}$ squared) in the tunnel (white square) and the cancellous bone around the tunnel (black square).
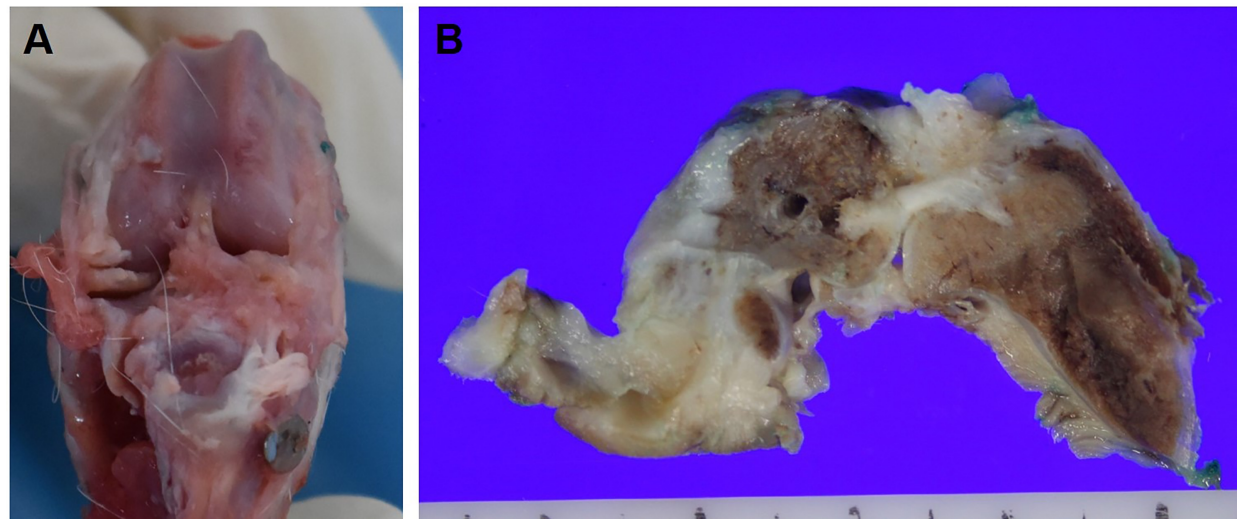

Fig. 3. (A) Gross photo and (B) oblique-sagittal section appearance showing reconstruced $\mathrm{ACL}$ on knee joint of rabbit.

section was stained with Hematoxylin and Eosin (H \& E). The stained slides of the knee joint sections were observed under a light microscope and were read by a pathologist.

\section{Statistical analysis}

Statistical evaluation was made using two ways repeated measures Kruskal-Wallis test to detect significant differences in the CT density scales and tunnel widening. When significant differences were found, post hoc comparisons with the Mann-Whitney test were made. The study results were evaluated with SPSS version 20.0 for Windows (SPSS Inc, Chicago, Illinois). Difference were considered statistically significant at $\mathrm{p}<0.05$.

\section{Results}

One rabbit in the control group died on postoperative 2 days and was excluded. One knee in the fibrin and one in the MSCs group had septic arthritis and were also excluded. The remaining 32 knees were evaluated radiologically and histologically. The average length of the grafts was $5.8 \mathrm{~cm}$ and all the grafts were intact at the time of dissection. Macroscopically, the implanted grafts were white in color with densely packed fiber bundles. All were cylindrical with a straight orientation.

\section{Radiologic findings}

The average combined length of the tunnels was $3.8 \mathrm{~cm}$. There were no statistically significant differences among the three groups in terms of tunnel lengths $(p>0.05)$.

Comparing the three groups, there are significant difference of femoral and tibial tunnel widening on the oblique-sagittal view ( $p=0.001,0.018$, respectively). The mean extent of widening of the femoral and tibial tunnel in oblique-sagittal image was significantly greater in the control than in the fibrin and MSCs groups $(\mathrm{p}<0.05)$ (Table 1). No significant differences were evident in terms of the mean tunnel widening on oblique-coronal views among 
Table 1. Tunnel widening following ACL reconstruction

\begin{tabular}{ccccc}
\hline & Control Group & fibrin Group & MSC Group & p-value* \\
\hline Femoral tunnel $(\mathrm{mm})$ & & & & $0.32 \pm 0.1$ \\
Oblique-coronal & $0.42 \pm 0.2$ & $0.27 \pm 0.1$ & $0.25 \pm 0.1$ & 0.288 \\
Oblique-sagittal & $0.70 \pm 0.4$ & $0.22 \pm 0.1$ & & 0.001 \\
Tibial tunnel (mm) & & & $0.44 \pm 0.3$ & 0.092 \\
Oblique-coronal & $0.65 \pm 0.3$ & $0.36 \pm 0.1$ & $0.29 \pm 0.2$ & 0.018 \\
Oblique-sagittal & $0.76 \pm 0.5$ & $0.27 \pm 0.1$ & \\
\hline
\end{tabular}

$\mathrm{ACL}$ : Anterior Cruciate Ligament, MSC: Mesenchymal Stem Cell, All values showed mean \pm SD.

${ }^{*} p$-value is statistically difference between the three groups (Kruskal-Wallis test).

Table 2. Hounsfield unit around the bone tunnel after ACL reconstruction

\begin{tabular}{|c|c|c|c|c|}
\hline & Control Group & fibrin Group & MSC Group & $p$-value* \\
\hline \multicolumn{5}{|l|}{ Femoral tunnel } \\
\hline In tunnel & $95.06 \pm 38.6$ & $103.69 \pm 21.8$ & $105.75 \pm 13.2$ & 0.248 \\
\hline Around tunnel & $424.88 \pm 70.0$ & $407.54 \pm 29.5$ & $387.30 \pm 50.1$ & 0.593 \\
\hline \multicolumn{5}{|l|}{ Tibial tunnel } \\
\hline In tunnel & $98.43 \pm 57.8$ & $102.77 \pm 32.4$ & $101.93 \pm 57.9$ & 0.797 \\
\hline Around tunnel & $405.85 \pm 55.2$ & $401.68 \pm 31.4$ & $421.08 \pm 42.7$ & 0.130 \\
\hline
\end{tabular}

ACL: Anterior Cruciate Ligament, MSC: Mesenchymal Stem Cell, All values showed mean \pm SD.

${ }^{*} p$-value is statistically difference between the 3 groups (Kruskal-Wallis test).

the three groups $(\mathrm{p}>0.05)$.

HUs in the femoral tunnel (control : $95.06 \pm 38.6$, fibrin : $103.69 \pm 21.8$, MSCs : $105.75 \pm 13.2)$ and in the tibial tunnel (control : $98.43 \pm 57.8$, fibrin : $102.77 \pm 32.4$, MSCs : $101.93 \pm 57.9)$ were similar to tendon structures in previous study (29). In addition, HUs around the femoral tunnel (control : 424.88 \pm 70.0 , fibrin : 407.54 \pm 29.5 , MSCs : $387.30 \pm 50.1$ ) and around the tibial tunnel (control : $405.85 \pm 55.2$, fibrin : 401.68 \pm 31.4 , MSCs : 421.08 \pm 42.7 ) were similar to prior values obtained from the cancellous bone of humans (29). HUs in and around the bone tunnel were not significantly different between the three groups $(\mathrm{p}>0.05)$ (Table 2).

\section{Histologic findings}

Gross observation revealed intact intra-articular grafts in all cases. For evaluation of tendon-bone integration and graft architecture, the intra-osseous portion of each graft was obtained and analyzed. $\mathrm{H} \& \mathrm{E}$ staining 12 weeks after surgery revealed preservation of smooth bone to graft bonding and good architecture were observed in the control group. However, there were fewer fibrocytes (hypocellularity) and less compact collagen fiber compared with those of other groups (Fig. 4A, D). Focal areas of disorganized architecture and degenerative alterations were evident in the control group. In the fibrin group, al- though disorganized architecture or partial tear of the graft was observed, there are good cellularity and more compact collagen fiber compared with the control group (Fig. 4B, E). In the MSCs group, excellent restoration of the bone to graft bonding to the well-formed tunnel was observed. The good architecture with hypercellularity and compact collagen deposition was shown in compared with other groups (Fig. 4C, F).

\section{Discussion}

The main purpose of this study was to compare the effect of MSCs and fibrin glue on ACL reconstruction in terms of radiologic evaluation as well as histologic assessment. The most important finding of present study was that MSCs seemed intra-articular graft healing and prevented tunnel widening.

Tendon-bone integration and graft healing after ACL reconstruction is a complex process. Natural tendon-bone integration without any biologic agent usually results in fibrous scar tissue, which is of inferior property compared to native attachment $(30,31)$. In addition, grafts undergo the process of avascular necrosis and revascularization the first period after their implantation $(32,33)$. This process lasts from 3 months to 1 year or more, and the detrimental effects of avascular necrosis on the mechanical properties 

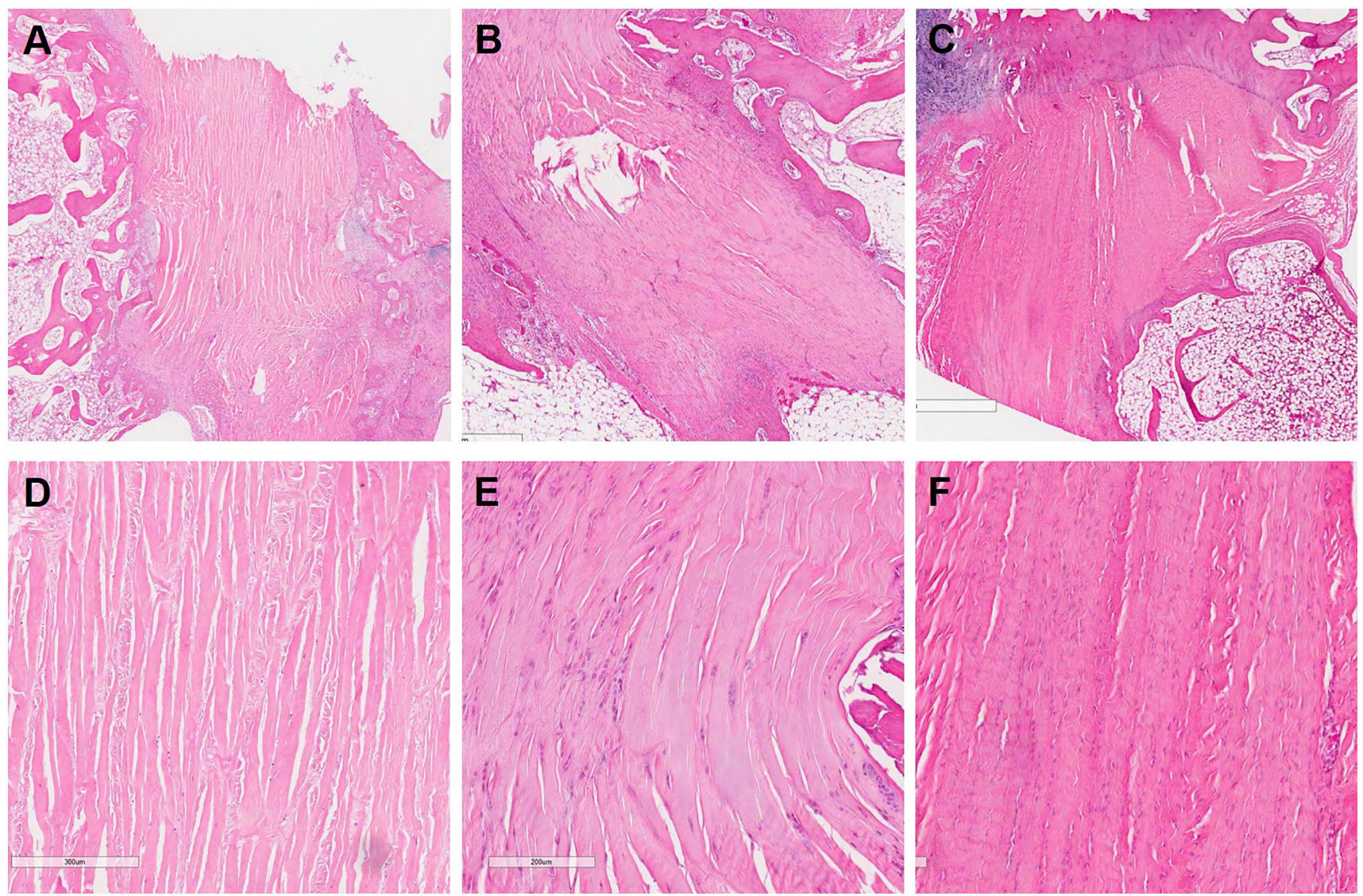

Fig. 4. Photomicrographs of $H \& E$ staining in 12-week $A C L$ reconstruction. In the control group $(A, D)$, the smooth tendon-bone integration (firm attachment fo the graft to the bone) were observed. However, hypocellularity and less compacted collagen fiber was observed. In fibrin group $(B, E)$, although partial tear of the graft has been shown, there are good cellularity and more compact collagen fiber. In the MSCs groups (C, F), the good architecture with hypercellularity and compacted collagen deposit was shown in compared with other groups.

can prelude graft failure or unsatisfactory function of the knee joint $(32,34,35)$.

For evaluation of the effect of MSCs on promoting graft osteointegration, Ouyang et al. (16) implanted bMSCs embedded within fibrin glue into the bone tunnel after ACL reconstruction in rabbit model. Four weeks later, Tendonbone interface revealed more perpendicular collagen fiber formation and increased proliferation of cartilage-like cells. Similar positive results were reported in two other studies using a rabbit model. These studies investigated the effect of MSCs on osteointegration of autografts (17) and allografts (18) in the bone tunnel. A mature zone from bone to the graft was observed in the MSCs group at 8 weeks in both studies, while the controls showed mature scar tissue resembling Sharpey's fibers spanning the tendon-bone interface.

The present experiment had demonstrated that administration of cultured MSCs caused an influx of cells into the ACL graft. Regarding the origin of these cells, one possibility is that the implanted MSCs proliferate and differentiate into ligament fibroblasts. Another possibility is that the cellular proliferation originates from cells recruited locally: injected MSCs may secrete a variety of growth factors to stimulate the activation and recruitment of local fibroblasts. An in vitro study on MSCs in graft healing reported that MSCs seemed to be the most suitable candidate for the development of tissue-engineered ligament, with the highest cell proliferation and highest collagen production (19).

Our study showed that the tunnel widening in the fibrin and MSCs groups was less than the control group after ACL reconstruction. Although whether tunnel widening influences clinical outcome is debatable, sagittal tunnel widening did positively affect on clinical outcome and anterior knee laxity after primary ACL reconstruction in one study (36).

There are several mechanical and biological factors for tunnel widening after ACL reconstruction. Biological fac- 
tors include inflammatory and immune response to allografts, increased cytokine levels within knee joint and cell necrosis caused by drilling of the bone $(37,38)$. In our experimental model, we tried to control mechanical factors including motion of the graft within the tunnel, insecure fixation, improper graft placement and accelerated rehabilitation. Simultaneously, we used MSCs and fibrin glue to prevent tunnel widening.

There was no difference in HUs between each group in the micro-CT analysis. This finding indicates that there was no heterotopic ossification in and around tunnels caused by MSCs. MSCs is likely to cause ectopic bone formation because of the multi-potential to differentiate into osteoblasts, chondrocytes and adipocytes. Actually, in a horse model, MSCs stimulated bone formation in the cartilage defects (39). However, no ectopic ossification was developed in and around the tunnels in the present study.

The rabbit ACL model has been validated in previous reports. Still, there are some limitations concerning the experimental model used in this study. A rabbit is too small for surgery and so differs from the human situation. Most studies of stem cells therapy are based on small animal models, in which the graft osteointegration process occurs at a faster rate than in humans. Further studies to test the effectiveness of this application in large animal models are necessary to confirm its clinical feasibility. In addition, more accurate and reliable experiments such as biomechanical testing are needed with a large animal model to best judge how to enhance the process of ligament reconstruction in humans. Lastly, we did not identify the ligamentization of the tendon using ligament-specific antibody by immunohistochemistry.

\section{Conclusion}

ACL reconstruction using MSCs seemed decrease tunnel widening and good graft architecture with hypercellularity and compact collagen deposition in the rabbit model. Further study with large animals is required to confirm efficacy on preventing tunnel widening.

\section{Acknowledgments}

All experimental animal procedures were according to the consent from the Institutional Animal Care and Use Committee of Chonnam National University Medical School (CNU IACUC-H-2015-33). This research was supported by the Bio \& Medical Technology Development Program of the NRF funded by the Korean government, MSIP (NRF-2016M3A9E9941832) and supported by Basic Science Research Program through the National Research
Foundation of Korea funded by the Ministry of Science, ICT \& Future Planning (NRF-017R1D1A3B03032027) and supported by a grant of National Research Foundation of Korea funded by the Ministry of Education, Science and Technology (NRF-2014R1A1A2059147).

\section{Potential Conflict of Interest}

The authors have no financial conflict of interest.

\section{References}

1. Spindler KP, Wright RW. Clinical practice. Anterior cruciate ligament tear. N Engl J Med 2008;359:2135-2142

2. Levine JW, Kiapour AM, Quatman CE, Wordeman SC, Goel VK, Hewett TE, Demetropoulos CK. Clinically relevant injury patterns after an anterior cruciate ligament injury provide insight into injury mechanisms. Am J Sports Med 2013;41:385-395

3. Sandberg R, Balkfors B, Nilsson B, Westlin N. Operative versus non-operative treatment of recent injuries to the ligaments of the knee. A prospective randomized study. J Bone Joint Surg Am 1987;69:1120-1126

4. Kaplan N, Wickiewicz TL, Warren RF. Primary surgical treatment of anterior cruciate ligament ruptures. A long-term follow-up study. Am J Sports Med 1990;18:354358

5. Strand T, Mølster A, Hordvik M, Krukhaug Y. Long-term follow-up after primary repair of the anterior cruciate ligament: clinical and radiological evaluation 15-23 years postoperatively. Arch Orthop Trauma Surg 2005;125:217221

6. Noyes FR, Butler DL, Grood ES, Zernicke RF, Hefzy MS. Biomechanical analysis of human ligament grafts used in knee-ligament repairs and reconstructions. J Bone Joint Surg Am 1984;66:344-352

7. Woo SL, Gomez MA, Seguchi Y, Endo CM, Akeson WH. Measurement of mechanical properties of ligament substance from a bone-ligament-bone preparation. J Orthop Res 1983;1:22-29

8. Melhorn JM, Henning CE. The relationship of the femoral attachment site to the isometric tracking of the anterior cruciate ligament graft. Am J Sports Med 1987;15:539-542

9. Muneta T, Yamamoto H, Sakai H, Ishibashi T, Furuya K. Relationship between changes in length and force in in vitro reconstructed anterior cruciate ligament. Am J Sports Med 1993;21:299-304

10. Kurosaka M, Yoshiya S, Andrish JT. A biomechanical comparison of different surgical techniques of graft fixation in anterior cruciate ligament reconstruction. Am J Sports Med 1987;15:225-229

11. Nikolaou VS, Efstathopoulos N, Wredmark T. Hamstring tendons regeneration after ACL reconstruction: an overview. Knee Surg Sports Traumatol Arthrosc 2007;15: $153-160$

12. Gulotta LV, Rodeo SA. Biology of autograft and allograft 
healing in anterior cruciate ligament reconstruction. Clin Sports Med 2007;26:509-524

13. Bissell L, Tibrewal S, Sahni V, Khan WS. Growth factors and platelet rich plasma in anterior cruciate ligament reconstruction. Curr Stem Cell Res Ther 2015;10:19-25

14. Vavken P, Sadoghi P, Murray MM. The effect of platelet concentrates on graft maturation and graft-bone interface healing in anterior cruciate ligament reconstruction in human patients: a systematic review of controlled trials. Arthroscopy 2011;27:1573-1583

15. Rodeo SA, Suzuki K, Deng XH, Wozney J, Warren RF. Use of recombinant human bone morphogenetic protein-2 to enhance tendon healing in a bone tunnel. Am J Sports Med 1999;27:476-488

16. Ouyang HW, Goh JC, Lee EH. Use of bone marrow stromal cells for tendon graft-to-bone healing: histological and immunohistochemical studies in a rabbit model. Am J Sports Med 2004;32:321-327

17. Lim JK, Hui J, Li L, Thambyah A, Goh J, Lee EH. Enhancement of tendon graft osteointegration using mesenchymal stem cells in a rabbit model of anterior cruciate ligament reconstruction. Arthroscopy 2004;20:899-910

18. Soon MY, Hassan A, Hui JH, Goh JC, Lee EH. An analysis of soft tissue allograft anterior cruciate ligament reconstruction in a rabbit model: a short-term study of the use of mesenchymal stem cells to enhance tendon osteointegration. Am J Sports Med 2007;35:962-971

19. Van Eijk F, Saris DB, Riesle J, Willems WJ, Van Blitterswijk CA, Verbout AJ, Dhert WJ. Tissue engineering of ligaments: a comparison of bone marrow stromal cells, anterior cruciate ligament, and skin fibroblasts as cell source. Tissue Eng 2004;10:893-903

20. Beyer Nardi N, da Silva Meirelles L. Mesenchymal stem cells: isolation, in vitro expansion and characterization. Stem Cells. Handb Exp Pharmacol 2006;(174):249-282

21. Oni OO. Early histological and ultrastructural changes in medullary fracture callus. J Bone Joint Surg Am 1992;74: 633-634

22. Pittenger MF, Mackay AM, Beck SC, Jaiswal RK, Douglas R, Mosca JD, Moorman MA, Simonetti DW, Craig S, Marshak DR. Multilineage potential of adult human mesenchymal stem cells. Science 1999;284:143-147

23. Awad HA, Boivin GP, Dressler MR, Smith FN, Young RG, Butler DL. Repair of patellar tendon injuries using a cell-collagen composite. J Orthop Res 2003;21:420-431

24. Li F, Jia H, Yu C. ACL reconstruction in a rabbit model using irradiated Achilles allograft seeded with mesenchymal stem cells or PDGF-B gene-transfected mesenchymal stem cells. Knee Surg Sports Traumatol Arthrosc 2007; 15:1219-1227

25. Atrah HI. Fibrin glue. BMJ 1994;308:933-934

26. Zazgyva AM, Gurzu S, Jung I, Nagy Ö, Mühlfay G, Pop TS. S53P4 bioactive glass and fibrin glue for the treatment of osteochondral lesions of the knee - a preliminary in vivo study in rabbits. Rom J Morphol Embryol 2015;56:10851090
27. Hao ZC, Wang SZ, Zhang XJ, Lu J. Stem cell therapy: a promising biological strategy for tendon-bone healing after anterior cruciate ligament reconstruction. Cell Prolif 2016; 49:154-162

28. Bachy M, Sherifi I, Zadegan F, Petrover D, Petite H, Hannouche D. Anterior cruciate ligament surgery in the rabbit. J Orthop Surg Res 2013 Aug 19 [Epub]. doi: 10.1186/1749-799x-8-27.

29. Gargiulo P, Helgason T, Reynisson PJ, Helgason B, Kern $\mathrm{H}$, Mayr W, Ingvarsson P, Carraro U. Monitoring of muscle and bone recovery in spinal cord injury patients treated with electrical stimulation using three-dimensional imaging and segmentation techniques: methodological assessment. Artif Organs 2011;35:275-281

30. Lu HH, Thomopoulos S. Functional attachment of soft tissues to bone: development, healing, and tissue engineering. Annu Rev Biomed Eng 2013;15:201-226

31. Pinczewski LA, Clingeleffer AJ, Otto DD, Bonar SF, Corry IS. Integration of hamstring tendon graft with bone in reconstruction of the anterior cruciate ligament. Arthroscopy 1997;13:641-643

32. Abe S, Kurosaka M, Iguchi T, Yoshiya S, Hirohata K. Light and electron microscopic study of remodeling and maturation process in autogenous graft for anterior cruciate ligament reconstruction. Arthroscopy 1993;9:394-405

33. Goradia VK, Rochat MC, Kida M, Grana WA. Natural history of a hamstring tendon autograft used for anterior cruciate ligament reconstruction in a sheep model. Am J Sports Med 2000;28:40-46

34. Blickenstaff KR, Grana WA, Egle D. Analysis of a semitendinosus autograft in a rabbit model. Am J Sports Med 1997;25:554-559

35. Grana WA, Egle DM, Mahnken R, Goodhart CW. An analysis of autograft fixation after anterior cruciate ligament reconstruction in a rabbit model. Am J Sports Med 1994;22: 344-351

36. Webster KE, Chiu JJ, Feller JA. Impact of measurement error in the analysis of bone tunnel enlargement after anterior cruciate ligament reconstruction. Am J Sports Med 2005;33:1680-1687

37. Vadalà A, Iorio R, De Carli A, Argento G, Di Sanzo V, Conteduca F, Ferretti A. The effect of accelerated, brace free, rehabilitation on bone tunnel enlargement after ACL reconstruction using hamstring tendons: a CT study. Knee Surg Sports Traumatol Arthrosc 2007;15:365-371

38. Cameron M, Buchgraber A, Passler H, Vogt M, Thonar E, $\mathrm{Fu} \mathrm{F}$, Evans $\mathrm{CH}$. The natural history of the anterior cruciate ligament-deficient knee. Changes in synovial fluid cytokine and keratan sulfate concentrations. Am J Sports Med 1997;25:751-754

39. Goodrich LR, Chen AC, Werpy NM, Williams AA, Kisiday JD, Su AW, Cory E, Morley PS, Mcllwraith CW, Sah RL, Chu CR. Addition of mesenchymal stem cells to autologous platelet-enhanced fibrin scaffolds in chondral defects: does it enhance repair? J Bone Joint Surg Am 2016;98:23-34 